\title{
Poster: Major Computational Breakthroughs in the Synthesis of Symbolic Controllers via Decomposed Algorithms
}

\author{
Eric S. Kim, Murat Arcak \\ Department of Electrical Engineering and Computer \\ Sciences, University of California \\ Berkeley, California, United States \\ eskim,arcak@eecs.berkeley.edu
}

\begin{abstract}
KEYWORDS
Symbolic Controllers, Finite Abstractions, Parallelization, Sparse Structures

ACM Reference Format:

Eric S. Kim, Murat Arcak and Mahmoud Khaled, Majid Zamani. 2018. Poster: Major Computational Breakthroughs in the Synthesis of Symbolic Controllers via Decomposed Algorithms. In HSCC '18: 21st International Conference on Hybrid Systems: Computation and Control (part of CPS Week), April 11-13, 2018, Porto, Portugal. ACM, New York, NY, USA, 3 pages. https://doi.org/10.1145/3178126.3187005
\end{abstract}

\section{INTRODUCTION}

Traditionally, control theory has been concerned with the basic stabilizability problem or the tracking of some reference signal. However, complex systems may require more sophisticated objectives such as those expressed in linear temporal logic or as automata on infinite strings. One popular approach to synthesize controllers that enforce complex specifications involves constructing finite abstractions that mimic the underlying continuous dynamics. Translating into a discrete domain opens up the ability to leverage tools from model checking and reactive synthesis. Tools such as [4], [6], and [3] implement abstraction and synthesis functionality for a wide variety of systems. The abstraction procedures in the aforementioned tools rely on partitioning the continuous state space and using reachable sets to construct transitions in the finite state machine. Controllers are synthesized by computing fixed points of a robust controllable predecessor operator. These tools have typically been limited to small ( $<5$ dimensional) systems because both the abstraction and synthesis steps suffer from acute time and space bottlenecks as the system dimension grows.

\subsection{Contributions}

Existing tools do no take advantage of the inherently parallelizable features of the abstraction and controller synthesis algorithms. We achieve orders of magnitude speedup by pursuing two independent approaches. First, we develop a parallelized core that takes

Permission to make digital or hard copies of part or all of this work for personal or classroom use is granted without fee provided that copies are not made or distributed for profit or commercial advantage and that copies bear this notice and the full citation on the first page. Copyrights for third-party components of this work must be honored.

For all other uses, contact the owner/author(s)

HSCC '18, April 11-13, 2018, Porto, Portugal

(C) 2018 Copyright held by the owner/author(s)

ACM ISBN 978-1-4503-5642-8/18/04.

https://doi.org/10.1145/3178126.3187005

\author{
Mahmoud Khaled, Majid Zamani \\ Department of Electrical and Computer Engineering, \\ Technical University of Munich \\ Munich, Germany \\ khaled.mahmoud,zamani@tum.de
}

advantage of independence and locality of abstraction and synthesis subroutines across different states. Second, we leverage the coordinate structure of the state space and sparsity in the system dynamics in order to eliminate unnecessary computations.

\section{PARALLELIZED CORE}

Transitions of abstract symbolic models can be computed independently and in parallel and fixed-point algorithms for controller synthesis also exhibit locality. Nevertheless, existing tools do not take advantage of such features. This leaves parallel computing resources, that are available today in almost any computing platform, underutilized. We present parallel implementations of the abstraction and synthesis phases that target hardware platforms with many (possibly distributed or clustered) processing elements.

\subsection{Parallel Construction of Symbolic Models}

For constructing symbolic models, each symbolic state is handled by a different processing unit. For each possible input to the system, the over-approximation of reachable sets is computed independently. According to available processing units, multiple symbolic states can share the same processing unit. Techniques like vectorization and pipelined execution are used to aggregate the execution of multiple states, leading to even more improvements in execution times. Subsets of the transitions in abstractions may also be stored in distributed memory, which provides flexibility in generating and accessing the abstractions' data.

\subsection{Parallel Synthesis of Symbolic Controllers}

We consider parallelizing fixed-point algorithms for the synthesis of symbolic controllers that satisfy invariance and reachability specifications. Several iterations that compute the Pre-map [4] are executed until the fixed-point set converges. Unlike the construction of symbolic models, the parallel computation of the Pre-map is not fully independent. To decide whether a state belongs to the fixed-point set or not, computations that depend on results from other states are essential. Having the memory of the fixed-point set distributed over multiple devices (or cores in same device), it is required to synchronize the computations and to collect the results after each iteration. We minimize the synchronization overhead by matching locality in cached memory to locality of dynamical systems (i.e., states depend on a group of states in the neighborhood).

\subsection{Implementation}

The abstraction and synthesis phases are both implemented in OpenCL, an open $\mathrm{C} / \mathrm{C}++$ standard for parallel programming. This 
Table 1: Comparison of the sequential and parallel implementations. Times include both abstractions and controller synthesis.

\begin{tabular}{llllll}
\hline & $\begin{array}{l}\text { Intel Core i7 } \\
(8 \text { cores })\end{array}$ & $\begin{array}{l}\text { Intel Xeon E5 } \\
(20 \text { cores })\end{array}$ & $\begin{array}{l}\text { Intel Core i7 (8 cores) } \\
\text { + Intel HD-530 GPU }\end{array}$ & $\begin{array}{l}\text { NVIDIA Quadro } \\
\text { K1200 (512 cores) }\end{array}$ & $\begin{array}{l}\text { NVIDIA Quadro } \\
\text { P5000 (2560 cores) }\end{array}$ \\
\hline $\begin{array}{l}\text { Parallel implementation } \\
\text { Abstraction + Synthesis Time (s) }\end{array}$ & 21 & 12 & 8.2 & 7.9 & 0.9 \\
\hline $\begin{array}{l}\text { Sequential implementation } \\
\begin{array}{l}\text { Abstraction + Synthesis Time (s) } \\
\hline\end{array}\end{array}$ & 210 & 153 & N/A & N/A & N/A \\
\hline
\end{tabular}

provides portable implementations that run on CPUs, GPUs, Hardware Accelerators and mixtures of them. To assess the speedup from the parallelization, we tested the initial implementations (still under improvement) with Intel CPUs, Intel GPUs and GPUs from NVIDIA. Table 1 shows our initial results for the autonomous vehicle example reported in [4], which easily exhibits two orders of magnitude improvement compared with sequential implementations. As a next step, we plan to target hardware accelerators like FPGAs, for which parallel implementations are deployed as hardware logic gates. We also plan for distributed implementations over the Cloud where clients (e.g., an autonomous vehicle) can request, on almost real-time, the synthesis of certifiable controllers to achieve some complex specifications.

\section{LEVERAGING SPARSITY TO ELIMINATE REDUNDANT COMPUTATIONS}

Many systems of large dimension are constructed via the composition of lower dimensional components. Existing abstraction and synthesis algorithms treat control systems as black boxes and ignore both the coordinate structure of the state space and independence in coordinate-wise updates in the system dynamics. Within the abstraction step, a full dimensional state traversal for a sparse control system will contain many redundant computations. Sparsity within the system dynamics is encapsulated in a dependency graph, which can be provided by a user or generated through syntactic analysis. By default, a system is assumed to contain an all-to-all dependency structure in which case the system is abstracted monolithically.

\subsection{Sparsity-Aware Abstraction}

The abstraction procedure can readily take advantage of sparsity annotations by selectively traversing lower dimensional grids. Unlike the brute force abstraction procedure, its sparsity-aware counterpart is linear with respect to state space and exponential with respect to a sparsity parameter that is upper bounded by the exponent in the monolithic case. Both the sparsity-aware and brute force abstraction procedures output the same result. This technique was used to abstract in minutes a 51-dimensional system representing a vehicular traffic network into a discrete system with $10^{63}$ states [1].

If the low dimensional grid traversal is still computationally prohibitive, one may also inject sparsity into the system by introducing additional hidden or latent variables. In effect, these variables reveal hidden sparsity by "lifting" the system dynamics into a higher dimensional, but simpler, representation. Sparsity-aware abstraction does not generally output the same result but instead yields a sound abstraction at the cost of additional spurious transitions [2].

\subsection{Decomposed Controllable Predecessor}

System dependencies are used to construct a computation graph that encodes a map from state-input pairs to their successive states. Computing a controllable predecessor can be interpreted as taking a set of outputted states and computing a functional pre-image. By analyzing the computation graph, it is possible to decompose the pre-image computation into smaller components which are systematically arranged to minimize memory overhead.

\subsection{Implementation}

The sparsity-aware abstraction and decomposed controllable predecessor have been implemented in a modified version of the SCOTS toolbox [4]. It makes extensive use of binary decision diagrams [5] to symbolically manipulate sets and systems. An open sourced implementation of the abstraction algorithm is available at https://github.com/ericskim/hscc18_modular. The decomposed controllable predecessor implementation is ongoing.

\section{COMBINING IMPLEMENTATIONS}

The above methods to respectively parallelize and decompose the controller synthesis procedure have been implemented separately. While different, the two approaches are compatible and current work is focused on merging the two implementations. The parallelized core will ensure that all available computational resources are utilized, whereas the sparsity-aware front end will reason about system dependencies to minimize redundant computations and communication across computing resources. We expect to easily obtain several orders of magnitude improvement in the computation time of the controller synthesis by combining both methods.

\section{REFERENCES}

[1] F. Gruber, E. Kim, and M. Arcak. 2017. Sparsity-Aware Finite Abstraction. In 2017 IEEE 56th Conference on Decision and Control (CDC).

[2] E. S. Kim, M. Arcak, and M. Zamani. 2018. Constructing Control System Abstractions from Modular Components. In Proceedings of the 21st International Conference on Hybrid Systems: Computation and Control (HSCC '18).

[3] S. Mouelhi, A. Girard, and G. Gössler. 2013. CoSyMA: A Tool for Controller Synthesis Using Multi-scale Abstractions. In 16th International Conference on Hybrid Systems: Computation and Control (HSCC '13). 83-88.

[4] M. Rungger and M. Zamani. 2016. SCOTS: A Tool for the Synthesis of Symbolic Controllers. In Proceedings of the 19th International Conference on Hybrid Systems: Computation and Control (HSCC '16). ACM, New York, NY, USA, 99-104.

[5] F. Somenzi. 2015. CUDD: CU Decision Diagram Package. http://vlsi.colorado.edu/ fabio/CUDD/. (2015). Version 3.0.0.

[6] T. Wongpiromsarn, U. Topcu, N. Ozay, H. Xu, and R. M. Murray. 2011. TuLiP: A Software Toolbox for Receding Horizon Temporal Logic Planning. In 14th International Conference on Hybrid Systems: Computation and Control (HSCC '11). 